\title{
Wiskott-Aldrich syndrome protein is required for regulatory $T$ cell homeostasis
}

\author{
Stephanie Humblet-Baron,, ${ }^{1,2}$ Blythe Sather,,3,4 Stephanie Anover, ${ }^{1}$ Shirly Becker-Herman, ${ }^{1}$ \\ Debora J. Kasprowicz, ${ }^{4}$ Socheath Khim, ${ }^{1}$ Thuc Nguyen, ${ }^{1}$ Kelly Hudkins-Loya, ${ }^{5}$ Charles E. Alpers, ${ }^{5}$ \\ Steve F. Ziegler, ${ }^{3,4}$ Hans Ochs, ${ }^{1}$ Troy Torgerson, ${ }^{1}$ Daniel J. Campbell, ${ }^{3,4}$ and David J. Rawlings ${ }^{1,3}$ \\ 1Department of Pediatrics, University of Washington School of Medicine, Seattle, Washington, USA. 2Center for Cellular and Molecular Therapy, \\ University of Liege, Liege, Belgium. ${ }^{3}$ Department of Immunology, University of Washington School of Medicine, Seattle, Washington, USA. \\ ${ }^{4}$ Benaroya Research Institute, Seattle, Washington, USA. ${ }^{5}$ Department of Pathology, University of Washington School of Medicine, Seattle, Washington, USA.
}

\begin{abstract}
Wiskott-Aldrich syndrome protein (WASp) is essential for optimal T cell activation. Patients with WAS exhibit both immunodeficiency and a marked susceptibility to systemic autoimmunity. We investigated whether alterations in Treg function might explain these paradoxical observations. While WASp-deficient (WASp ${ }^{-/-}$) mice exhibited normal thymic Treg generation, the competitive fitness of peripheral Tregs was severely compromised. The total percentage of forkhead box P3-positive $\left(\mathrm{Foxp}^{+}\right)$Tregs among $\mathrm{CD}^{+} \mathrm{T}$ cells was reduced, and $\mathrm{WASp}^{-/-}$Tregs were rapidly outcompeted by WASp${ }^{+}$Tregs in vivo. These findings correlated with reduced expression of markers associated with self-antigen-driven peripheral Treg activation and homing to inflamed tissue. Consistent with these findings, WASp ${ }^{-/-}$Tregs showed a reduced ability to control aberrant $\mathrm{T}$ cell activation and autoimmune pathology in Foxp $3^{---}$Scurfy $(s f)$ mice. Finally, WASp ${ }^{+}$Tregs exhibited a marked selective advantage in vivo in a WAS patient with a spontaneous revertant mutation, indicating that altered Treg fitness likely explains the autoimmune features in human WAS.
\end{abstract}

\section{Introduction}

Wiskott-Aldrich syndrome (WAS) is an X-linked immunodeficiency disorder characterized by opportunistic, viral, and bacterial infections due to abnormal lymphocyte function. Affected individuals also have thrombocytopenia with small platelets, eczema, and increased risk of autoimmune disorders and malignancies (1). Worldwide, more than 200 unique mutations have been described within the gene encoding the WAS protein (WASp) (reviewed in ref. 2). Mutations leading to loss of WASp expression correlate with a more severe disease phenotype (3). WASp mRNA is expressed in all hematopoietic lineages, and WASp participates in multiple signal transduction pathways in a range of cell types. WASp deficiency, however, is most prominently associated with defects in Tlymphocyte function. WASp $\mathrm{W}^{-/} \mathrm{T}$ cells fail to polymerize and reorganize actin in response to anti-CD3 stimulation, and formation of the $\mathrm{T}$ cell immunological synapse is defective. Thus, WASp deficiency appears to directly interfere with assembly of the TCR "signalosome," resulting in incomplete cellular activation and, consequently, decreased cell proliferation and cell survival.

Patients with WAS exhibit a very high prevalence of autoimmune disease. In one study more than $70 \%$ of patients $(40 / 55$ evaluated) had 1 or more autoimmune episodes, including autoimmune cytopenias, arthritis, vasculitis, inflammatory bowel disease, or renal disease $(1,4)$. Autoimmune manifestations in WAS typically present very early in life and are largely unresponsive to medical therapy. In addition, WAS patients with autoimmune disease manifest a poorer clinical prognosis (3). Further, even patients with otherwise mild disease (thrombocytopenia only)

Nonstandard abbreviations used: CFSE, carboxy-fluorescein diacetate, succinimidyl ester; DN, double negative; dsDNA, double-stranded DNA; Foxp3, forkhead box P3; sf, Scurfy; SP, single positive; ssDNA, single-stranded DNA; WAS, Wiskott-Aldrich syndrome; WASp, WAS protein.

Conflict of interest: The authors have declared that no conflict of interest exists. Citation for this article: J. Clin. Invest. 117:407-418 (2007). doi:10.1172/JCI29539. due to mutations permitting low-level expression of intact protein or of a partially functional protein can develop life-threatening autoimmune sequelae (3). The high prevalence of autoimmunity in WAS might result from escape of self-reactive $T$ cells from negative selection due to defective TCR-mediated signals and reduced apoptosis at this checkpoint. Alternatively, chronic inflammatory stimuli, defects in IL-2 production, or alterations in APC or macrophage function have each been suggested as explanations of these disease associations $(5,6)$. In this study, we tested to determine whether these paradoxical observations, immunodeficiency in association with life-threatening autoimmunity, might be explained on the basis of defects in Treg function and altered dominant tolerance.

Naturally occurring $\mathrm{CD} 4^{+}$Tregs mediate dominant control of self-reactive $\mathrm{T}$ cells and thereby play a crucial role in maintenance of immunologic self tolerance (reviewed in refs. 7,8 ). Tregs are generated in the thymus, and the development of this population is critically dependent upon expression of the forkhead box transcription factor, forkhead box P3 (Foxp3), which serves as the best marker to identify both thymic and peripheral Tregs. Recent evidence indicates that the TCR repertoire of Tregs is likely to be as broad as that of other naive T cells, enabling Tregs to recognize both self and nonself antigens. While much has been learned with regard to the generation and functional properties of Tregs, relatively less is known with regard to their in vivo activation, including, in particular, the specific signals that control Treg expansion and tissue localization. Such signals are likely to be crucial in mediation of the distinct downstream regulatory activities of activated Tregs.

We show that $\mathrm{WASp}^{-/-}$mice, like WAS patients, develop early onset, high-titer autoantibodies. We also show that restoration of WASp expression in humans promotes the expansion of Tregs. Consistent with both of these observations, WASp ${ }^{-1-}$ Tregs fail to compete effectively in vivo and are unable to maintain immunologic 


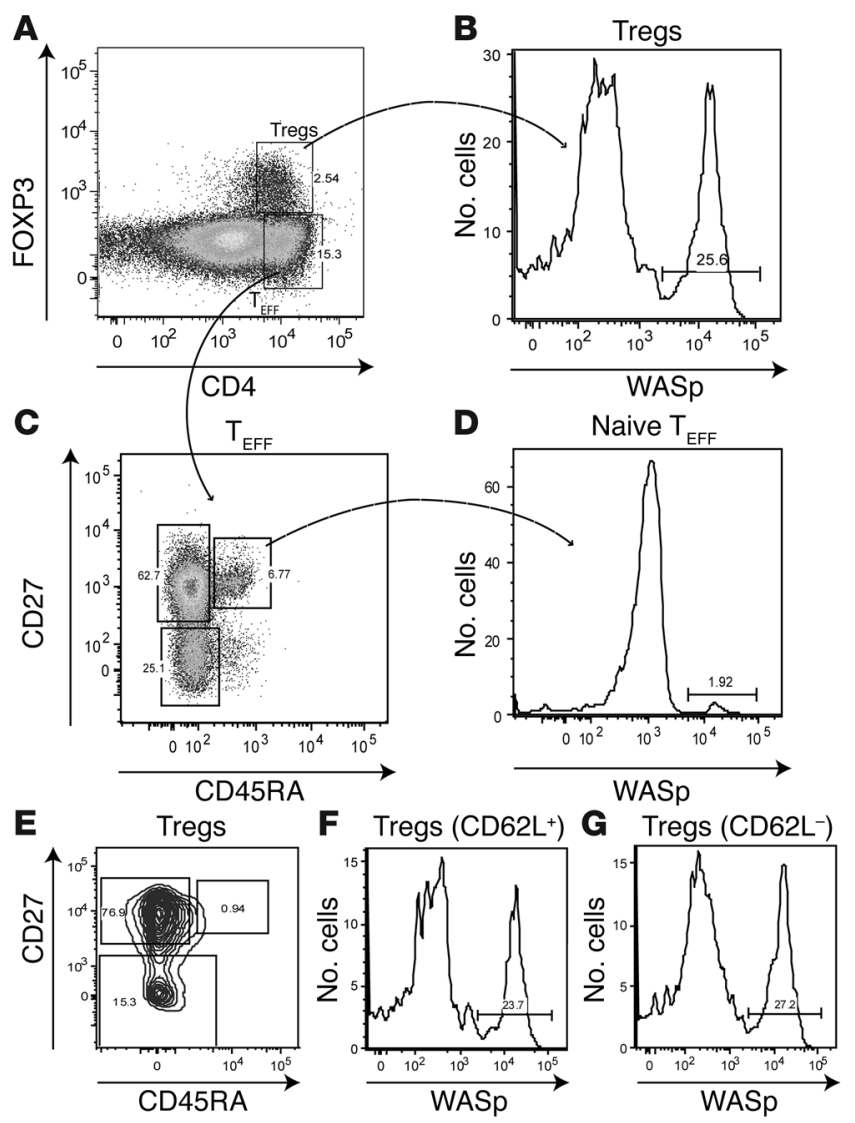

tolerance in Treg-deficient mice. Finally, we show that the percentage of Tregs expressing adhesion molecules and chemoattractant receptors required for nonlymphoid tissue entry is uniformly reduced in $\mathrm{WASp}^{-/-}$Tregs, suggesting a defect in peripheral Treg activation. Taken together, our findings indicate that Treg homeostasis is critically reliant upon signals integrated by WASp and suggest a crucial role for WASp in antigen-driven Treg expansion and control of basal $\mathrm{T}$ and $\mathrm{B}$ cell activation in normal hosts.

\section{Results}

Expansion of differentiated WAS $p^{+}$Tregs in a WAS patient with a revertant mutation. We recently identified a $\mathrm{WASp}^{-/-}$patient who exhibited an improved clinical picture in association with new evidence for WAS expression within his T, B, and NK cells. This teenaged patient had suffered from lifelong, recurrent episodes of autoimmune hemolytic anemia beginning at 15 months of age. WASp reexpression correlated with stabilization of his $\mathrm{rbc}$ count and reduction in his steroid therapy over the preceding 6-9 months. Previous diagnostic studies had identified a singlenucleotide deletion in WAS leading to a frameshift and premature stop codon as well as absence of WASp expression. These genetic studies were repeated using peripheral blood lymphocytes and a newly derived $T$ cell line and identified a new singlenucleotide insertion at the same genomic site. This change was predicted to restore the normal amino acid sequence and normal WASp expression (T. Torgerson, H. Ochs, and D.J. Rawlings, unpublished observations).

This revertant mutation provided a unique opportunity to evaluate the consequences of restored WASp function in newly

\section{Figure 1}

WT Tregs are expanded in a WAS patient following reversion of a pathogenic mutation. Peripheral blood mononuclear cells were analyzed by flow cytometry using antibodies to CD4, CD45RA, CD27, CD62L, WASp, and FOXP3. (A) Characterization of the CD4 ${ }^{+} \mathrm{FOXP}^{+}$ (Tregs) and $\mathrm{CD}^{+}{ }^{+} \mathrm{FOXP3}^{-}$(effector $\mathrm{T}$ cell $\left[\mathrm{T}_{\mathrm{EFF}}\right]$ ) cell populations within the total lymphocyte gate. (B) WASp expression within the $\mathrm{CD}^{+}{ }^{+} \mathrm{FXP}^{+}$Treg population demonstrating that approximately $25 \%$ of the patient's Tregs are WASp ${ }^{+}$. (C) Identification of the naive CD $4{ }^{+} \mathrm{CD} 27{ }^{+} \mathrm{CD} 45 \mathrm{RA}+{ }^{+} \mathrm{T}$ cells within the CD4+FOXP3 $\mathrm{T}_{\text {EFF }}$ cell population. Naive cells account for approximately $7 \%$ of the $T_{E F F}$ population in this patient. (D) Within the naive T cell compartment, only a small pro-

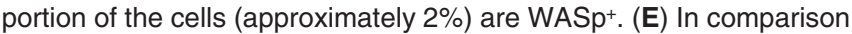
with the $T_{\text {EFF }}$ population, very few $(<1 \%)$ CD27+CD45RA ${ }^{+}$naive cells are present within the $\mathrm{CD}^{+}{ }^{+} \mathrm{FOXP} 3^{+}$Treg population. ( $\mathbf{F}$ and $\left.\mathbf{G}\right)$ Within the $\mathrm{CD}^{+}{ }^{+} \mathrm{FOXP}^{+}$Treg population, WASp ${ }^{+}$cells account for equal percentages of the $\mathrm{CD}^{2} \mathrm{~L}^{+}(\mathbf{F})$ and $\mathrm{CD} \mathrm{L}^{-}(\mathbf{G})$ Treg subsets.

generated lymphoid populations. Consistent with the reversion having an impact on a limited progenitor pool, only approximately $2 \%$ of naive $\mathrm{CD} 4 \mathrm{~T}$ cells (e.g., $\mathrm{CD} 4{ }^{+} \mathrm{CD} 45 \mathrm{RA}^{+} \mathrm{CD} 27^{+} \mathrm{CD} 62 \mathrm{~L}^{+}$ cells) expressed WASp (Figure 1, C and D). In contrast, we observed a striking increase in relative percentage of WASp ${ }^{+}$ Tregs (25\%-35\% CD4 ${ }^{+} \mathrm{FOXP}^{+}$cells expressed WASp; Figure 1B). Similar results were obtained in 3 independent analyses over time. To define the relative expression of WASp within maturing Tregs, we also used markers to identify CD45RA ${ }^{+} \mathrm{CD} 27^{+}$, $\mathrm{CD}^{2} 5 \mathrm{RA}^{-} \mathrm{CD} 27^{+}$, and CD45RA-CD27- Tregs (Figure 1E). Based upon these staining criteria, very few $\mathrm{CD} 45 \mathrm{RA}^{+} \mathrm{CD} 27^{+}$naive cells were present within the $\mathrm{CD} 4^{+} \mathrm{FOXP} 3^{+}$Treg population. We also evaluated the expression of CD62L within the $\mathrm{CD}^{+}{ }^{+} \mathrm{FOXP} 3^{+}$ Treg population. WASP ${ }^{+}$cells accounted for $24 \%$ and $27 \%$ of the CD45RA-CD62 $\mathrm{L}^{+}$and $\mathrm{CD}^{-} 45 \mathrm{RA}^{-} \mathrm{CD} 62 \mathrm{~L}^{-}$subsets, respectively (Figure 1, F and G). In contrast, CD45RA $\mathrm{CD}^{+} 2 \mathrm{~L}^{+}$Tregs accounted for less than $1 \%$ of the total Treg population, and the very small number of such cells precluded analysis of the relative percentage of $\mathrm{WASp}^{+}$cells within this naive population (due to the lymphopenia present in this patient). These results suggest that WASp ${ }^{+}$human Tregs manifested a strong in vivo selective advantage and raise the question of whether WASp plays a critical role in Treg homeostasis.

WASp ${ }^{-/-}$mice develop bigh-titer anti-DNA autoantibodies and autoimmune disease. WASp-deficient $\left(\operatorname{WASp}^{-/-}\right)$mice $(9,10)$ provide a useful model for human WAS. These animals exhibit clear defects in $\mathrm{T}$ cell function, including abnormal actin cytoskeletal organization, reduced CD3 and CD3/CD28 proliferative responses, and markedly reduced IL-2 production. Despite the striking clinical data in human WAS, however, there is only limited evidence that $\mathrm{WASp}^{-/-}$mice develop autoimmunity. The 129SvEv/ $\mathrm{WASp}^{-/-}$strain (9) develops spontaneous, radiation-induced colitis that resembles autoimmune inflammatory bowel disease. We have also observed spontaneous subclinical colitis and frequent rectal prolapse in $\mathrm{WASp}^{-/-}$mice backcrossed into the $\mathrm{C} 57 \mathrm{BL} / 6$ background (data not shown). Thus, this inflammatory disease association, while less severe, does not appear to be strain specific as previously suggested (11).

Notably, no previous studies have evaluated whether WASp $\mathrm{WA}^{-/}$ mice exhibit defects in B cell tolerance or develop humoral autoimmune features analogous to those commonly observed in patients with WAS. To address this question, we initially screened a cohort of aged $\mathrm{WASp}^{-/}$and control animals for evi- 
A
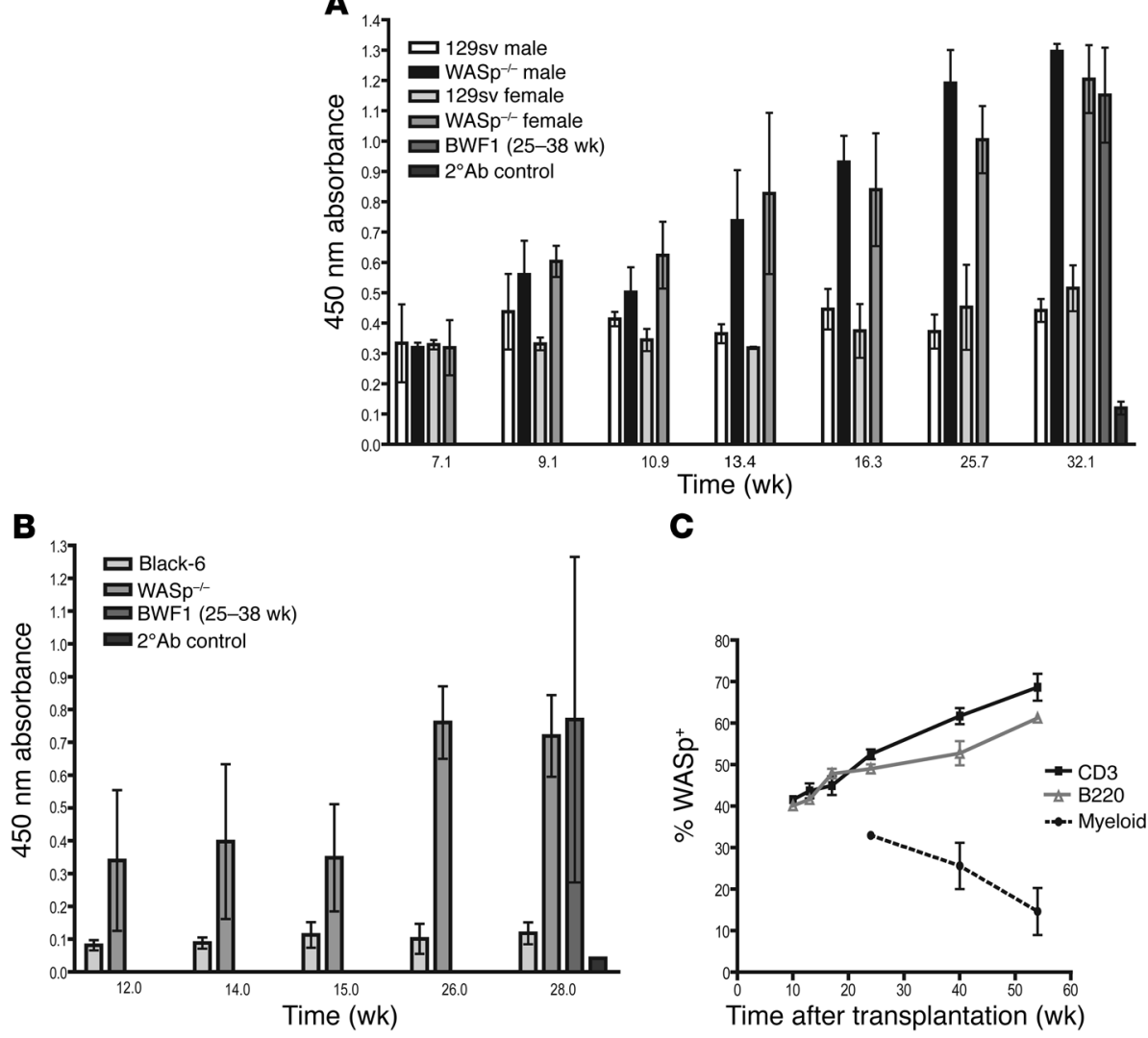

C

D
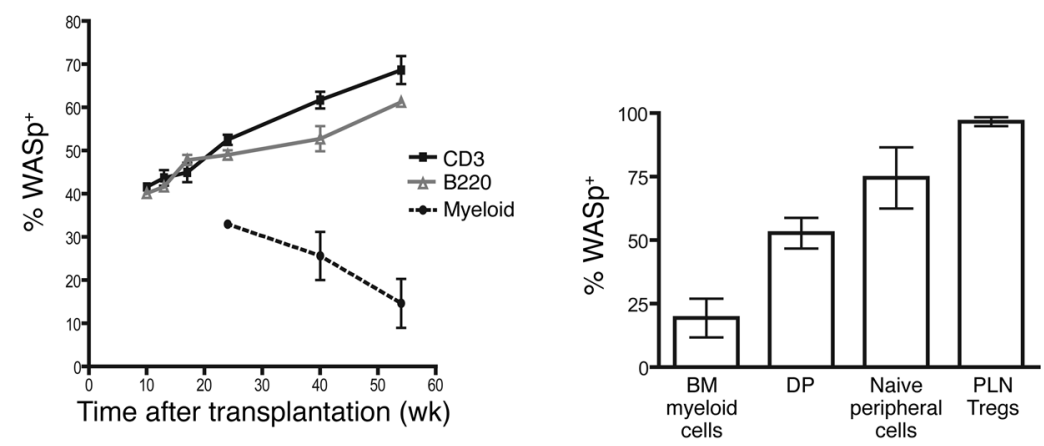

Figure 2

WASp ${ }^{-/-}$mice develop high-titer autoantibodies, and WT Tregs expand and rescue transplanted WASp ${ }^{-/-}$mice from autoimmune colitis. Presence of IgG dsDNA autoantibodies in the serum of (A) 129SvEv (129sv) or (B) C57BL/6 (Black-6) WASp ${ }^{-/-}$mice versus age- and sex-matched WT controls or 25- to 38-week-old BWF1 mice (positive control). Antibody titers were assessed by ELISA ( $n=3-6$ animals for each strain/sex). $2^{\circ} \mathrm{Ab}$ control, background values obtained using secondary antibody alone. Error bars represent SD. (C) WASp expression within T and B cells (CD3 and B220, respectively) but not myeloid cells allows for a competitive advantage over time in lethally irradiated WASp ${ }^{-1-}$ recipients (129SvEv strain) transplanted with a 1:3 mixture of WT to WASp ${ }^{-1-}$ BM cells. Peripheral blood from 5 mice was serially analyzed by flow cytometry at the indicated times after transplant to determine the percentage of WASp ${ }^{+} \mathrm{T}, \mathrm{B}$, or myeloid cells. (D) The selective advantage of WASpexpressing cells within the T cell compartment is most marked in the peripheral Treg subset. WASp ${ }^{-/-}$mice (129SvEv strain) were transplanted as in C, and WASp expression among the indicated T cell populations was evaluated 12 months after transplant. WASp ${ }^{+}$myeloid cells remain at the same percentage as when originally transplanted (approximately $25 \%$ ), indicating no selective advantage. Recipients of WT:WASp ${ }^{-/-}$mixed $\mathrm{BM}$ transplants did not develop fatal, radiation-induced colitis, which occurred in all recipients of WASp ${ }^{-1-} \mathrm{BM}$ (data not shown). DP, CD4+CD8+ thymocytes; PLN Tregs, peripheral lymph node Tregs.

dence of antinuclear antibodies and observed a marked increase in both anti-single-stranded DNA (anti-ssDNA) and anti-double-stranded DNA (anti-dsDNA) antibodies (Supplemental Figure 1A; supplemental material available online with this article; doi:10.1172/JCI29539DS1). To determine the timing and frequency of autoantibody production, we followed cohorts of $\mathrm{WASp}^{-/-}$and control mice of both sexes and of both genetic backgrounds. Compared with age-matched WT controls, WASP-/mice demonstrated a consistent increase in both anti-dsDNA and anti-histone antibodies by 3 months of age (Figure 2, A and B, and Supplemental Figure 1B). Significantly, titers of anti-DNA antibodies in 6-month-old $\mathrm{WASP}^{-/-}$mice were equivalent to those in 6- to 9-month-old female NZB/W F F $_{1}$ mice, a well-characterized murine model of systemic lupus erythematosus (12). Together, our data demonstrate that WAS $\mathrm{W}^{-/}$mice develop high-titer antinuclear antibodies with high frequency early in life, indicating that WASp deficiency promotes alterations in B cell tolerance.
Chimeric BM transplantation promotes WT Treg expansion and rescues WASP $\mathrm{p}^{-1-}$ from irradiation-induced colitis. Irradiation promotes the rapid onset of inflammatory colitis in $\mathrm{WASp}^{-/}$mice (129SvEv strain). This complication is prevented by transplantation with WT but not WASp ${ }^{-/-}$bone marrow. For example, 11 of $12 \mathrm{WASp}^{-/-}$ animals developed severe colitis requiring sacrifice following radiation doses of 550-950 cGy and transplantation with $\mathrm{WASp}^{-/-} \mathrm{BM}$ whereas no animals transplanted with WT BM developed this complication (data not shown). Because of these observations, we decided to determine whether induction of colitis in this model was due to a cell-intrinsic defect in tolerance induction of the WASp $^{-/-}$cells. To this end, bone marrow $\left(1 \times 10^{7}\right.$ containing a 1:3 chimeric mixture of WT versus $\mathrm{WASp}^{-/-}$cells) was transplanted into lethally irradiated $129 \mathrm{SvEv} \mathrm{WASp}^{-/-}$mice to establish stable mixed chimeras. In lieu of congenic markers, we utilized WASp intracellular staining as a highly sensitive assay to identify WASp ${ }^{+}$ and $\mathrm{WASp}^{-/-}$hematopoietic cells. Recipient animals exhibited 
A

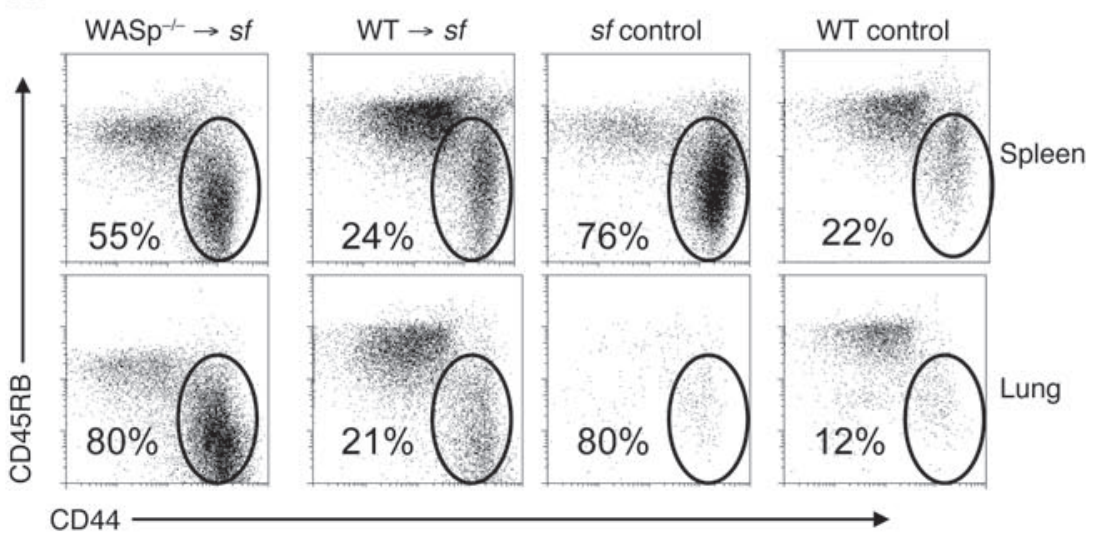

B

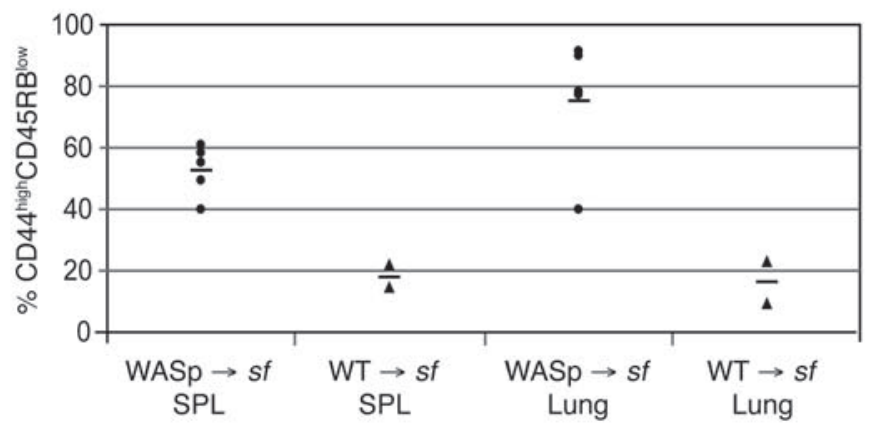

C
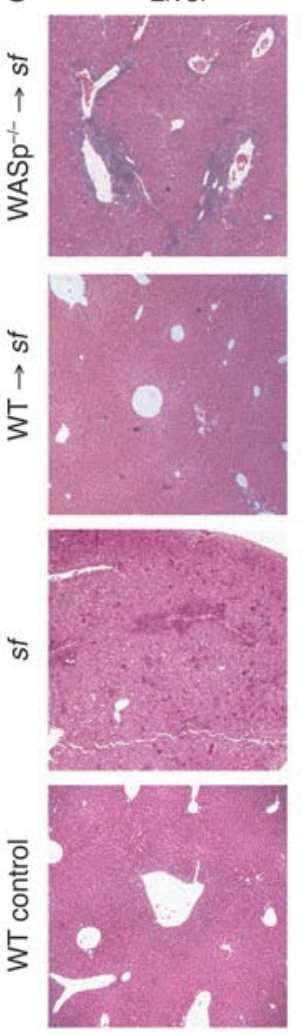

Lung
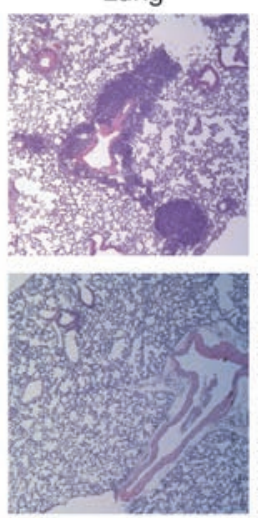
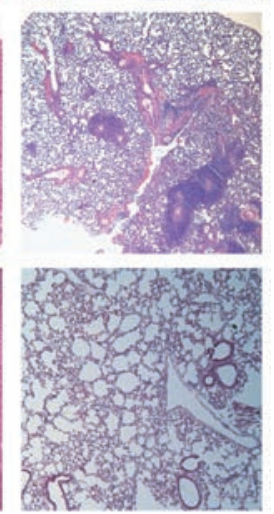

\section{Figure 3}

WASp $^{-1-}$ Tregs fail to control autoimmunity in sf mice. Male Ly5.1 sf neonates (>3 days of age) were injected i.p. with $1 \times 10^{6}$ to $2 \times 10^{6}$ CD4 ${ }^{+}$CD25 $5^{+}$-enriched WT or WASp ${ }^{-/-}$Tregs (both Ly5.2), sacrificed at 30-45 days after cell transfer, and evaluated for levels of T cell activation and tissue inflammation. (A) WT Tregs but not WASp ${ }^{-/}$Tregs prevent development of activated sf lymphocytes. Lymphocytes isolated from the spleen and lung parenchyma of recipient mice were stained for CD4, Ly5.1, CD44, and CD45RB. The relative percentage of activated CD44hi CD45RB ${ }^{\text {low }} T$ cells in each tissue is shown. All plots are gated on CD4+Ly5.1+ cells to identify recipient-derived cells, and donor cell source is indicated above each panel. Controls included age-matched, unmanipulated sf and WT animals. (B) Graph represents the percentage of CD44hiCD45RB low-activated, recipient-derived cells (CD4+Ly5.1+) among all recipient animals. Each point represents data from 1 sf recipient of either WASp ${ }^{--}(n=5)$ or WT $(n=2)$ Tregs. (C) WT Tregs but not WASp ${ }^{-1}$ Tregs rescue sf mutant mice from development of autoimmune infiltration of major organs. Formalin-fixed liver and lung tissue from sf mice that received WT versus WASp ${ }^{-1-}$ Tregs were paraffin embedded, sectioned, and stained with H\&E to visualize tissue structure and inflammatory cell infiltration. Liver and lung sections from unmanipulated sf and WT mice are shown for comparison. Original magnification, $\times 10$.

a progressive accumulation of $\mathrm{WASp}^{+} \mathrm{B}$ and $\mathrm{T}$ cells (reaching approximately $40 \%$ at 10 weeks and $65 \%-75 \%$ by 50 weeks after transplantation; Figure 2C). Similar findings were previously reported (11) and indicate that WASp expression provides a selective advantage to both $\mathrm{T}$ and $\mathrm{B}$ cells under these conditions. Interestingly, while detailed phenotypic analysis of $\mathrm{T}$ cell subsets indicated only limited selection for WASp ${ }^{+}$cells within the thymus and naive $\mathrm{T}$ cell pool, we observed a preferential expansion of WASp ${ }^{+}$ Tregs. The relative numbers of WASp ${ }^{+}$Tregs reached nearly $100 \%$ at the time of sacrifice (12 months after transplantation; Figure 2D). In contrast, we observed no advantage for WASp $\mathrm{W}^{+}$myeloid cells $\left(\mathrm{Gr}-1^{+}\right.$and $\mathrm{Mac}^{+}$cells), which were maintained at levels equivalent to the initial chimeric mixture (25\%-30\%; Figure $2 \mathrm{C}$ and data not shown). Notably, none of the transplanted mice developed overt signs of colitis, and histological analysis at the time of sacrifice was indistinguishable from that for age-matched WT controls (data not shown). Thus, WT cells ameliorated the colitis mediated by $\mathrm{WASp}^{-/-}$cells, and this correlated with a preferential expansion of WASp $^{+}$Tregs within $\mathrm{WASp}^{-/-}$hosts.
Transfer of WASp ${ }^{-/-}$Tregs fails to control autoimmunity in neonatal Scurfy recipient mice. Because $\mathrm{WASP}^{+}$effector $\mathrm{T}$ cells and $\mathrm{B}$ cells were also partially selected in vivo in our chimeric bone marrow transplant experiments, these studies were insufficient to determine whether defects in Treg function were principally responsible for the autoimmune features observed in $\mathrm{WASp}^{-/-}$mice. Male Scurfy (sf) mice lack all Tregs due to a spontaneous mutation in Foxp3 and suffer from severe multiorgan autoimmunity, resulting in death by 3-4 weeks of age. Adoptive transfer of even very small numbers of WT Tregs $\left(\sim 10^{5}\right)$ can prevent the sf autoimmune syndrome (7). Therefore, we used this model to directly test for cell-intrinsic in vivo defects in $\mathrm{WASp}^{-/-}$Treg function independent of any contribution of functional defects in WASp $\mathrm{W}^{-/}$ effector T cells. Male sf pups received purified WT Tregs (as a control for prevention of autoimmunity) or $\mathrm{WASp}^{-/-}$Tregs via adoptive transfer. In each case, donor Tregs (Ly5.2) were transferred into nonirradiated C57BL/6 (Ly5.1/Ly5.2 heterozygous) neonatal $s f$ recipients. As previously described, unmanipulated $s f$ animals exhibited severe lymphadenopathy, splenomegaly, and 
A

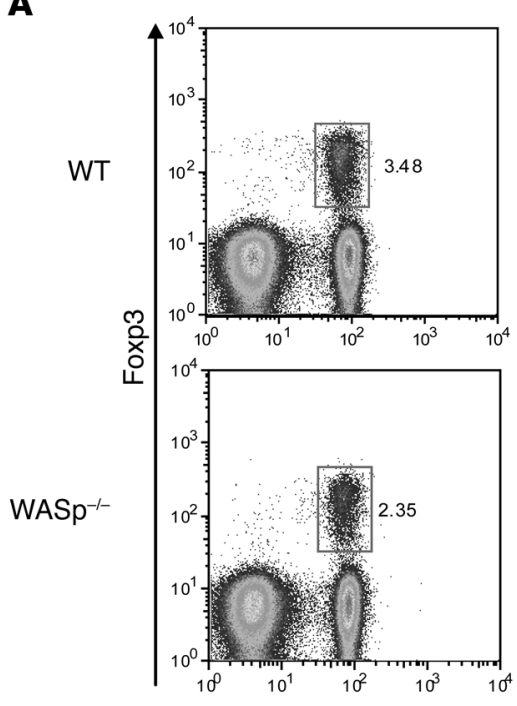

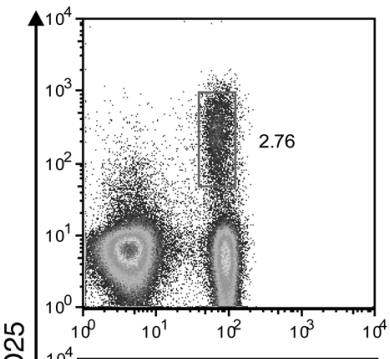

กิ่

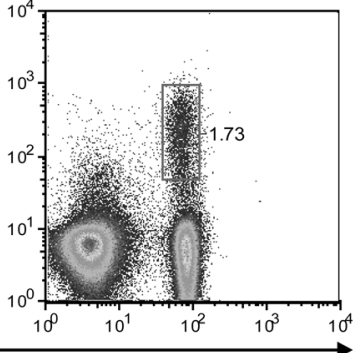

CD4

C

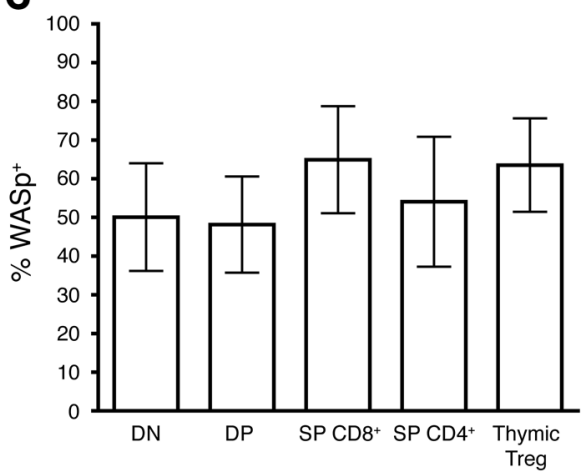

\section{Figure 4}

WASp is not required for generation of Tregs within the thymus. (A) WASp is not required for the production of peripheral Tregs. Peripheral LN cells from WT or WASp ${ }^{-1-}$ animals were stained simultaneously for CD4, CD25, and Foxp3 and evaluated by flow cytometry. Note that Foxp $3^{+}$ Tregs are present in WASp ${ }^{-/}$mice albeit at a slightly decreased percentage. (B) WASp ${ }^{-1-}$ and WT mice have a similar percentage of Tregs (CD4+ $\mathrm{CD}^{+} 5^{+} \mathrm{Foxp}^{+}$) within the CD4+ $\mathrm{SP}$ thymic population. Both 6- and 16week-old WT and WASp ${ }^{-1-}$ C57BL/6 mice ( $n=5$ for each age and strain) were evaluated. (C) The selective advantage of WASp ${ }^{+} \mathrm{T}$ cells is not manifest in the thymus. The percentage of $\mathrm{WASp}^{+}$cells was evaluated within various thymic cell subsets in 6to 8-week-old WASp $\mathrm{W}^{+/}$heterozygous female carriers (C57BL/6 strain) $(n=5)$. Error bars show SD. Relative WASp expression was not significantly different among any subset evaluated. DN, CD4-CD8- thymocytes. Representative data from 1 of at least 3 experiments are shown. phenotypic evidence for $\mathrm{T}$ cell activation and autoimmunity in all tissues examined. $\mathrm{CD}^{+} \mathrm{T}$ cells isolated from the spleen and lung parenchyma expressed elevated levels of the activation marker CD44 and reduced expression of the naive $\mathrm{T}$ cell marker CD45RB (Figure 3A). Histological analysis of liver and lung, 2 particularly susceptible tissues in $s f$ mice, revealed extensive lymphocytic infiltration and inflammation surrounding blood vessels in the liver and large and small airways in the lung (Figure 3C). In contrast, animals receiving adoptively transferred WT Tregs exhibited minimal T cell activation (Figure 3, A and B) and little or no histological evidence of lymphocytic inflammation in all tissues analyzed (lung and liver shown, Figure 3C). These findings were similar to those in unmanipulated WT control mice (Figure 3, A and C). Strikingly, recipients of $\mathrm{WASp}^{-/-}$Tregs were unable to control aberrant activation of effector T cells (Figure 3, A and B) and developed marked splenomegaly and pulmonary and liver inflammatory changes that were similar to those in untreated $s f$ mice (Figure 3C). Inflammatory cell infiltrates were also observed in other tissues, including skin and kidney (data not shown). Importantly, all Ly5.2+ cells were uniformly Foxp $3^{+}$, indicating that the transferred cell populations were composed entirely of either $\mathrm{WASp}^{-/-}$or WASP $\mathrm{WA}^{+}$ Tregs, respectively (data not shown). Taken together, these data demonstrate that $\mathrm{WASp}^{-/-}$Tregs fail to effectively mediate dominant tolerance in vivo.
WASP ${ }^{-1-}$ mice generate normal numbers of Foxp $3^{+}$Tregs within the thymus. Based upon the in vivo functional deficit exhibited by WASp $\mathrm{WA}^{-/}$ Tregs, we next sought to determine whether WASp deficiency had an impact specifically on either Treg generation, in vitro Treg function, or in vivo homeostasis. To address the role of WASp in Treg production, we identified Tregs in WT versus WASp $\mathrm{WA}^{-/-}$ animals using 2 independent staining protocols (Figure 4A). Tregs were readily identified in $\mathrm{WASp}^{-/}$mice as based on either the $\mathrm{CD}^{+} \mathrm{CD}^{4}{ }^{\text {int }} \mathrm{CD} 25^{\text {hi }} \mathrm{CD} 69^{-}$or $\mathrm{CD} 4 \mathrm{Foxp} 3^{+}$cell phenotype. There was no difference in either the relative percentage or absolute numbers of $\mathrm{CD}^{+} \mathrm{Foxp}^{+}$cells in the thymi of WT versus $\mathrm{WASp}^{-/-}$mice in either the C57BL/6 or $129 \mathrm{SvEv}$ strains (Figure 4B and data not shown).

To more precisely assess the role for WASp thymic Treg production, we evaluated WASp expression in thymic T cell subsets isolated from WASp $\mathrm{P}^{+/-}$heterozygous female mice. This approach allowed us to identify any developmental stage(s) at which WAS $\mathrm{p}^{+}$cells might manifest a selective advantage based upon nonrandom inactivation of the X-linked WAS gene. One previous study has suggested that BM progenitor cells derived from $\mathrm{WASp}^{+/-}$heterozygous mice exhibit early nonrandom $\mathrm{X}$ inactivation (13). However, intracellular WASp staining of both BM B cell progenitors and myeloid cells from heterozygous animals was consistent with a random X-inactivation pattern, with $50 \%$ of cells in both lineages expressing WASp (Supplemental Figure 2A 


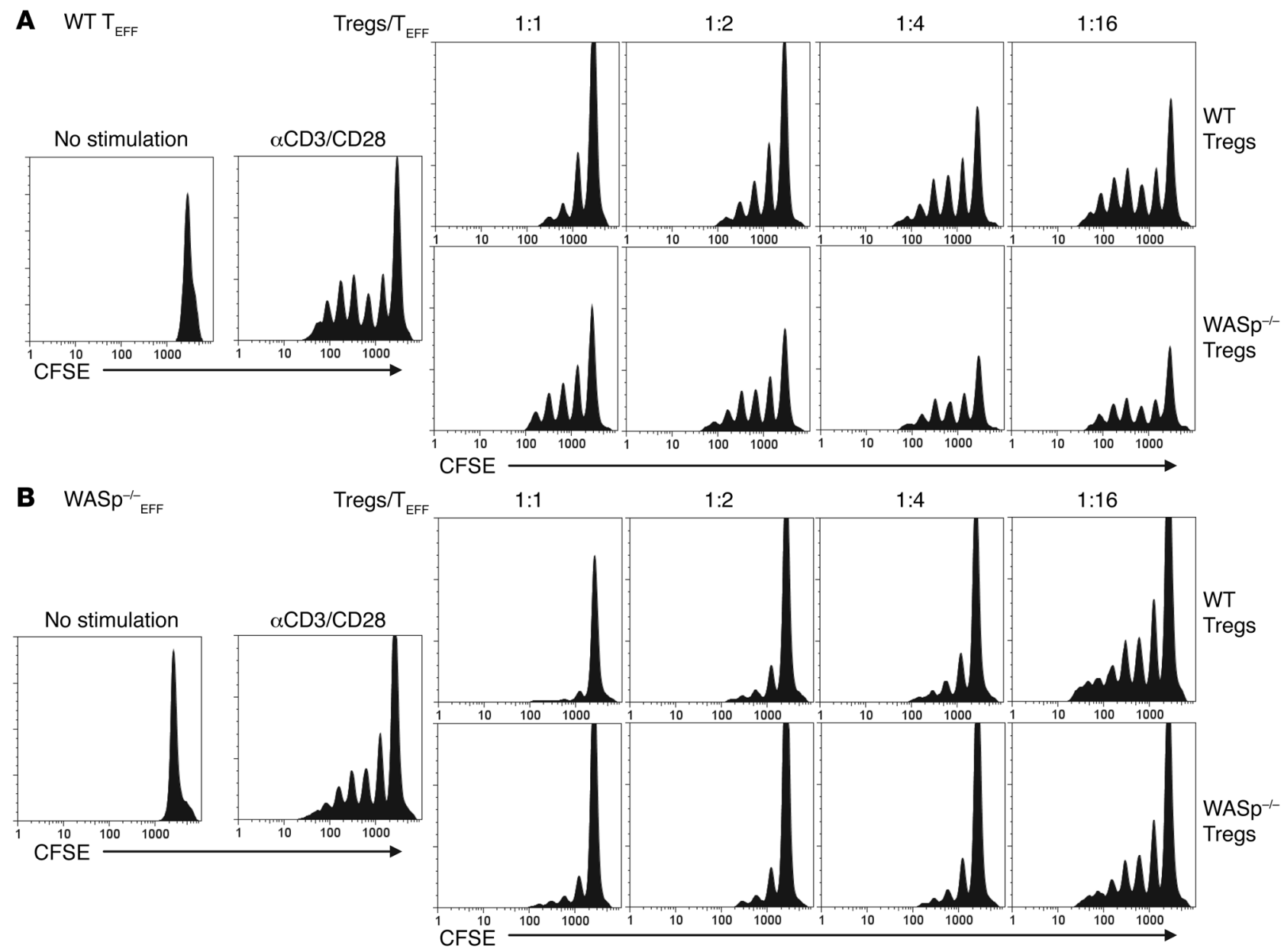

Figure 5

WASp ${ }^{-1-}$ Tregs exhibit in vitro suppressive activity. CD4 ${ }^{+} \mathrm{CD} 25^{-}$effector $\mathrm{T}$ cells $\left(\mathrm{T}_{\mathrm{EFF}}\right)$ and $\mathrm{CD} 4^{+} \mathrm{CD} 25^{+}$cells (Tregs) were isolated from WT or $\mathrm{WASp}^{-/-}$mice (C57BL/6 strain). WT or WASp $/-\mathrm{T}_{\mathrm{EFF}}$ were labeled with CSFE and plated as targets with WT or WASp $/-$ Tregs at the Treg/T (target) ratios noted in the presence of irradiated APCs. Cultures were stimulated with $3 \mu \mathrm{g} / \mathrm{ml}$ anti-CD3 and $1 \mu \mathrm{g} / \mathrm{ml}$ anti-CD28 for 110 hours. Relative CFSE dilution was measured in cultures containing WT (A) or WASp ${ }^{-1-}$ (B) targets. Unstimulated and control-stimulated (CD3/CD28 without Tregs) cells are shown in the left panels.

and data not shown). Our data suggest that previous findings may reflect a selective advantage of $\mathrm{WASp}^{+}$cells for growth in colony-forming assays. The relatively limited role of WASp in murine versus human HSC function might reflect redundant activity of neural WASp or related proteins.

Using this staining method, we observed little or no difference in the relative percentage of $\mathrm{WASp}^{+}$cells within any thymic developmental stage, from double negative (DN) to single positive (SP). The median number of WASp $\mathrm{P}^{+}$cells increased slightly between the DN and SP stages, reaching a level of slightly greater than $50 \%$ in $\mathrm{CD}^{+}$and $\mathrm{CD}^{+}{ }^{+} \mathrm{SP}$ thymocytes (Figure $4 \mathrm{C}$ ). While a previous report (10) suggested that WASp deficiency impairs the double-negative 3 (DN3) to DN4 transition, we also observed no significant change in the relative percentage of WASp-expressing cells at this stage (data not shown). Most notably, the median level for WASp expression in thymic Foxp $3^{+}$Tregs was essentially identical to that of the total $\mathrm{CD} 4^{+} \mathrm{SP}$ thymocytes in young (6-8.5 weeks) and aged (6 months) heterozygous mice (Figure 4C and data not shown). Thus, WASp is not essential for generation of Tregs in the thymus.
WASp ${ }^{-/-}$Tregs exhibit in vitro suppressive activity. We used in vitro T cell suppression assays to directly determine whether WASp was required for Treg-suppressive activity. Spleen and $\mathrm{LN} \mathrm{CD} 4^{+} \mathrm{CD} 25^{-}$ effector $\mathrm{T}$ cells were labeled with carboxy-fluorescein diacetate, succinimidyl ester (CSFE) or left unlabeled, then cultured with increasing numbers of WT or WASp ${ }^{-/-} \mathrm{CD} 4^{+} \mathrm{CD} 25^{+}$Tregs. All cultures also contained irradiated CD4-depleted WT APCs and were stimulated with agonistic anti-CD3 and anti-CD28 monoclonal antibodies. When tested with WT effector T cells, WASp ${ }^{-/-}$Tregs exhibited moderately reduced suppressor activity at all target ratios, based upon either CSFE dilution or thymidine incorporation as independent measures for effector proliferation (Figure 5A and data not shown). To better mimic the in vivo situation in $\mathrm{WASp}^{-/-}$mice, we also evaluated the relative suppressor activity of WT versus $\mathrm{WASp}^{-/-}$Tregs with respect to $\mathrm{WASp}^{-/-}$effectors. Under these conditions (Figure 5B), WT and WASp ${ }^{-/-}$Tregs functioned equivalently at all target ratios. Additionally, we compared the suppressive activity of WT versus WASp $\mathrm{W}^{-/}$Tregs across a range of anti-CD3 cross-linking to determine whether WASp 

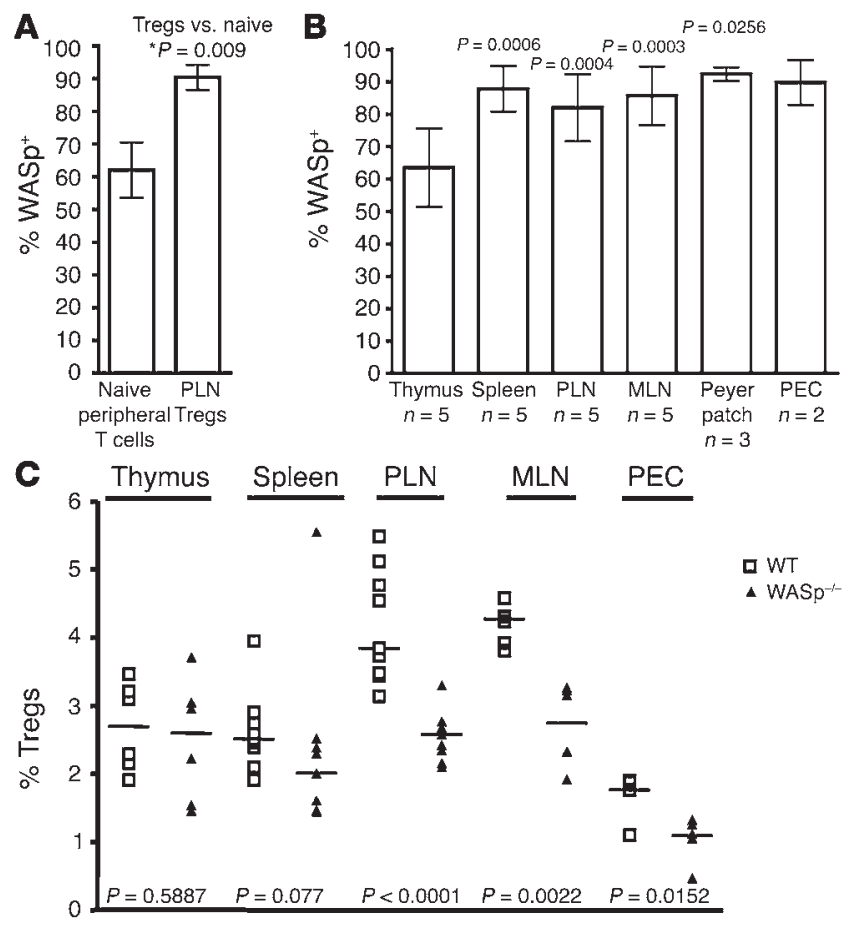

deficiency impaired Treg function in the context of suboptimal CD3 engagement. Again, WT and $\mathrm{WASp}^{-/-}$Tregs functioned similarly at each CD3 concentration evaluated (data not shown; Treg/effector $\mathrm{T}$ cell ratio of 1:1). Together, these findings indicate that, in contrast with the marked in vivo functional deficit observed following adoptive transfer, Treg activation and function in vitro are largely intact in the absence of WASp. Further, because WASp ${ }^{-/-}$Tregs efficiently suppressed $\mathrm{WASp}^{-/-}$effectors, alterations in Treg function appeared insufficient to explain the autoimmune phenotype of WASp-deficient mice or humans.

$W$ SP $^{-1-}$ Tregs fail to compete effectively in vivo. While WASp was not required for the generation or in vitro activity of Tregs, we reasoned that defects in Treg homeostasis might account for the failure of $\mathrm{WASp}^{-/-}$Tregs to control self reactivity in vivo. To address this issue, we first analyzed the relative numbers of $\mathrm{WASp}^{+}$versus $\mathrm{WASp}^{-/-}$Tregs within peripheral lymphatic tissues of WAS $\mathrm{p}^{+/-}$heterozygous female mice. An example of this analysis is provided in Supplemental Figure 2B. While only approximately $55 \%$ of naive T cells were WASp ${ }^{+}$, more than $90 \%$ of peripheral Tregs were $\mathrm{WASp}^{+}$(Figure 6A; $P<0.009$ ). This skewing was obvious by 6 weeks of age, maintained in older animals, and evident in all lymphoid tissues as well as in cells isolated from nonlymphoid sites, including the peritoneal cavity (peritoneal cavity exudate cells) and lung airways (bronchoalveolar lavage fluid cells) (Figure 6B and data not shown).

We also determined whether the apparent selective advantage of WT Tregs observed in heterozygous WAS $\mathrm{p}^{+/-}$mice was reflected in alterations in the relative numbers of Tregs in $\mathrm{WASp}^{-/-}$animals (Figure 6C). While the frequency of thymic Tregs was not different in 6-, 16-, and 24-week-old WT versus $\mathrm{WASp}^{-/}$animals, the percentage of $\mathrm{CD}^{+}{ }^{+} \mathrm{Foxp} 3^{+}$Tregs was consistently reduced in all peripheral lymphoid compartments in $\mathrm{WASp}^{-/}$animals. This reduction was present in mice at all ages evaluated in both C57BL/6 and 129SvEv WASP-/- strains (Figure 6C and data not

\section{Figure 6}

$\mathrm{WASp}^{-1-}$ Tregs demonstrate a competitive disadvantage in vivo. (A) Heterozygous female carriers (6 months) demonstrate marked skewing within the Treg population. Naive $T$ cells from peripheral blood $\left(\mathrm{CD}^{+} \mathrm{CD} 62 \mathrm{~L}^{+} \mathrm{CD} 44^{-}\right)$and Tregs from peripheral $\mathrm{LNs}$ $\left(C D 4{ }^{+} \mathrm{CD} 25^{+}{ }^{+}\right.$D $\left.69-\right)$ were analyzed for relative WASp expression by flow cytometry. Mean \pm SD is shown with the results of the paired 2-tailed Student's $t$ test. Identical results were obtained using $\mathrm{CD}^{+}{ }^{+} \mathrm{Foxp}^{+}$staining to identify Tregs. PEC, peritoneal cavity exudate; MLN, mesenteric lymph node. (B) WASp confers selective advantage during peripheral maturation/expansion of Tregs. The Treg population in different tissues from 6- to 8-week-old WASp ${ }^{+/}$heterozygous mice (C57BL/6 strain) was evaluated for WASp expression by flow cytometry gated on the CD4+Foxp3 ${ }^{+}$population. Paired 2-tailed Student's $t$ test results indicate significant differences between peripheral lymphoid and thymic Tregs. (C) WASp ${ }^{-/-}$mice have lower Treg numbers than WT mice. CD4+Foxp3+ Tregs were evaluated in different tissues in WT versus $\mathrm{WASp}^{-/} \mathrm{C} 57 \mathrm{BL} / 6$ mice (ages 6 weeks to 6 months) and displayed as relative percentage of Tregs within the live cell gate. Pooled data were compared using the Mann-Whitney $U$ test.

shown). Because $\mathrm{WASp}^{-/-}$mice also exhibit a modest reduction in total $\mathrm{CD} 4^{+} \mathrm{T}$ cell numbers, this change resulted in an approximate $30 \%$ reduction in the number of total $\mathrm{CD}^{+}{ }^{+}$Foxp $3^{+}$splenic Tregs compared with WT animals. Notably, we observed no significant differences in relative percentage(s) of $\mathrm{CD}^{+}, \mathrm{CD}^{+}$, or $\mathrm{CD}^{+}$cells within the peripheral lymphatic compartment compared with controls. This finding was consistent with a preferential loss of Tregs within the peripheral $\mathrm{T}$ cell pool in $\mathrm{WASp}^{-/}$animals.

Purified WASp $\mathrm{P}^{-1-}$ Tregs expand poorly in neonatal recipient mice. Homeostatic maintenance of Tregs in nonlymphopenic hosts is dependent upon both TCR signals and exogenous IL-2 provided via $\mathrm{CD}^{+}{ }^{+} \mathrm{CD} 25^{\text {low }}$ Foxp3 $3^{-} \mathrm{T}$ cells $(14,15)$. Exogenous IL-2 levels are predicted to be decreased in $\mathrm{WASp}^{-/}$mice due to the deficit in TCR-mediated IL-2 production $(9,10)$, and this might limit the expansion/function of WASp ${ }^{-/-}$Tregs. To test this possibility, WT (Ly5.1) and WASp ${ }^{-/-}$(Ly5.2) Tregs were isolated and cotransferred $\left(2 \times 10^{6}\right.$ to $6 \times 10^{6}$ total Tregs/recipient $)$ into nonirradiated, neonatal C57BL/6 (Ly5.1/Ly5.2) sf recipients. Upon transfer, purified WT Tregs undergo extensive homeostatic expansion (7). Thus, by cotransferring WT and $\mathrm{WASp}^{-/-}$Tregs into neonatal $s f$ mice, we could directly compare their ability for homeostatic expansion and survival in the context of normal IL-2 production and in the absence of endogenous Tregs. Adoptively transferred Tregs were identified using antibodies to the Ly5.1 (CD45.1) versus Ly5.2 (CD45.2) allotype markers beginning at 14 days after transfer (Figure $7 \mathrm{~A}$ ). WASp $\mathrm{W}^{-/}$Tregs were rapidly outcompeted by the cotransferred WT Tregs, indicated by their progressive decline from spleen and LNs within 20-30 days after transfer (Figure 7, A and B). Thus, IL-2 provided in trans by normal effector $\mathrm{T}$ cells does not appear to be sufficient to rescue homeostatic proliferation of $\mathrm{WASp}^{-/-}$Tregs.

Impaired peripheral differentiation of $\mathrm{WASP}^{-/-}$Tregs. The majority of Tregs undergo cell division following transfer into nonlymphopenic hosts $(14,15)$, and proliferating Tregs in naive animals acquire a distinct activated cell surface phenotype, including downmodulation of CD62L and increased expression of CD44, CD69, and other activation markers (15). To determine whether WASp may play an important role in Treg activation, we asked whether $\mathrm{WASp}^{+}$versus $\mathrm{WASp}^{-/-}$Tregs derived from $\mathrm{WAS}^{+/-} \mathrm{car}^{-}$ riers differed with regard to expression of these markers. WAS $\mathrm{p}^{+}$ 
A
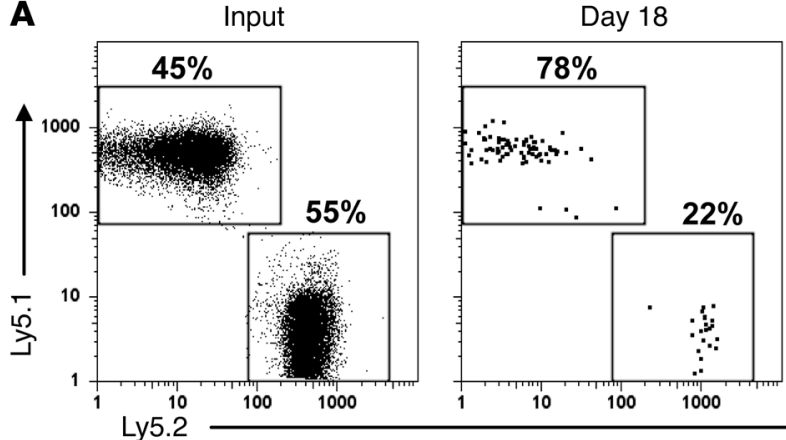

B

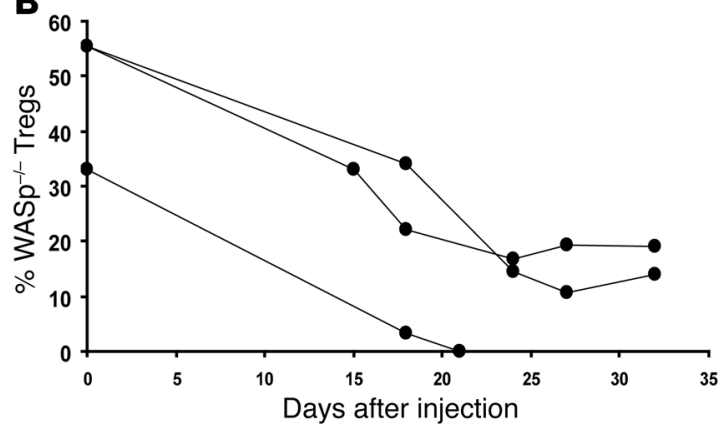

Day 27

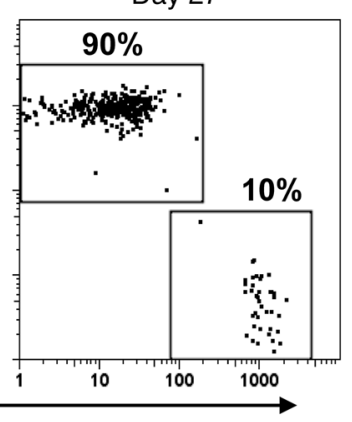

\section{Figure 7}

Purified WASp ${ }^{-/-}$Tregs fail to expand and compete effectively in vivo. Male sf neonates (>4-5 days of age) heterozygous for Ly5.1 and Ly5.2 were injected i.p. with a 50:50 mixture of WT (Ly5.1) and WASp ${ }^{-1-}$ (Ly5.2) CD4 ${ }^{+} \mathrm{CD} 25^{+}$Tregs. Peripheral blood lymphocyte samples were analyzed at biweekly intervals starting at 14 days of age to measure the relative levels of donor Tregs. (A) Representative temporal analysis of the relative numbers of WT versus WASp ${ }^{-1-}$ Tregs. FACS analysis of input (left panel) CD4 ${ }^{+}$CD25+-enriched WT/WASp ${ }^{-/-}$Treg mixture stained for Ly5.1 and Ly5.2 (middle and right panels). Analysis of the relative level for each donor population within the $\mathrm{CD}^{+}{ }^{+} \mathrm{Foxp}^{+}$gate at time points indicated. Peripheral blood lymphocyte samples were costained for CD4, Foxp3, Ly5.1, and Ly5.2. (B) Graphic depiction of the relative percentage of WASp ${ }^{-1}$ Tregs remaining at each time point in 3 animals, based upon phenotypic analysis as described in $\mathbf{A}$.
Tregs consistently expressed higher levels of both CD44 and CD69 and reduced levels of CD62L (data not shown). Essentially identical results were obtained using either $C D 4^{\text {int }} \mathrm{CD} 25^{\text {hi }}$ or $\mathrm{CD}^{+}{ }^{+} \mathrm{Foxp}^{+}$gates (as in Figure 4A) to define Treg subsets. These findings suggest that Treg activation preferentially promoted the expansion of WASp ${ }^{+}$cells.

Notably, Tregs are thought to be targeted to nonlymphoid sites only after self-antigen recognition within lymphoid tissues. Thus, Tregs have been subdivided based on homing receptor expression into populations with differential tropism for lymphoid versus nonlymphoid tissues (reviewed in ref. 16). Tregs expressing the $\alpha \mathrm{E}$ chain (CD103) of the integrin CD103 $\beta 7$ also preferentially express homing receptors that target cells to nonlymphoid tissues, including P selectin and E selectin ligands, $\beta 1$ integrin, CCR4, and CCR6 $(17,18)$. While $\mathrm{CD}_{103^{-}}$and $\mathrm{CD} 103^{+}$Tregs exhibit similar in vitro suppressor activities, they exhibit the distinct capacity to suppress in vivo immune responses. Naive $\mathrm{CD}^{+} \mathrm{T}$ cell activation in lymphoid tissues is effectively suppressed by CD103- Tregs, whereas $\mathrm{CD}_{103}{ }^{+}$Tregs home to inflammatory sites and preferentially suppress disease activity in inflammatory models $(18,19)$ or modulate the effector $\mathrm{T}$ cell response to local infection (20).

To determine the relative requirement for WASp in generation of tissue-tropic Treg subsets, we characterized cell surface expression profile of WASp ${ }^{+}$versus WASp- Tregs with regard to a series of candidate adhesion and homing receptors. Essentially identical data were obtained in analysis of Tregs isolated from spleen or from peripheral or mesenteric LNs (Figure 8 and data not shown). CCR7 is expressed by the vast majority of Tregs in lymphoid tissues, and accordingly, we observed no appreciable difference in the relative expression of this receptor on WASp ${ }^{+}$versus $\mathrm{WASp}^{-/-}$Tregs (Figure 8D). In contrast, both the relative percentage (Figure 8, C-E) and relative mean fluorescence intensity of CD103 expression (data not shown) were significantly reduced in WASP- Tregs. Accordingly, the relative expression of $\mathrm{P}$ selectin and $\mathrm{E}$ selectin ligands, CCR4, and
CCR6 were also significantly reduced in $\mathrm{WASp}^{-/-}$Tregs (Figure 8E). Although the number of cells available for analysis was relatively limited, we also observed an increase in CD103 expression in $\mathrm{WASp}^{+}$Tregs isolated from bronchoalveolar lavage fluid and peritoneal cavity exudate (data not shown). Together, this phenotypic analysis supports the idea that WASp-dependent signals are required for optimal activation and functional differentiation of Tregs in the periphery.

\section{Discussion}

The data presented here strongly support a model in which signals mediated by WASp are essential for Treg homeostasis, peripheral activation, and in vivo function. While WASp exerts little or no role in thymic Treg production, WASp is required for peripheral Treg expansion and survival. WASp deficiency leads to a decreased percentage of Tregs among the peripheral $\mathrm{CD} 4^{+} \mathrm{T}$ cells and has a marked impact on the activated Treg pool, as shown by the decrease in Tregs that express activation markers and homing receptors associated with activation. Consistent with these observations, $\mathrm{WASp}^{-/-}$Tregs exhibited decreased competitive fitness in 3 independent in vivo murine models, including heterozygous female carriers, recipients of chimeric bone marrow transplants, and Treg-deficient mice that received $\mathrm{WASp}^{-/-}$Tregs via adoptive transfer. Further, expansion of WASp ${ }^{+}$Tregs correlated with rescue of $\mathrm{WASp}^{-/-}$recipients from radiation-induced inflammatory colitis, and conversely, WASp ${ }^{-/-}$Tregs failed to mediate dominant tolerance after transfer into neonatal $s f$ mice. Finally, consistent with each of these observations in mice and with the marked propensity for WAS patients to develop autoimmune sequelae, we observed a strong selective advantage for $\mathrm{WASp}^{+}$human Tregs in vivo in a patient with a revertant mutation leading to reexpression of WAS in developing Tregs. In this case, the presence of this population correlated with decreased autoimmune disease activity and an improved clinical condition. 
A
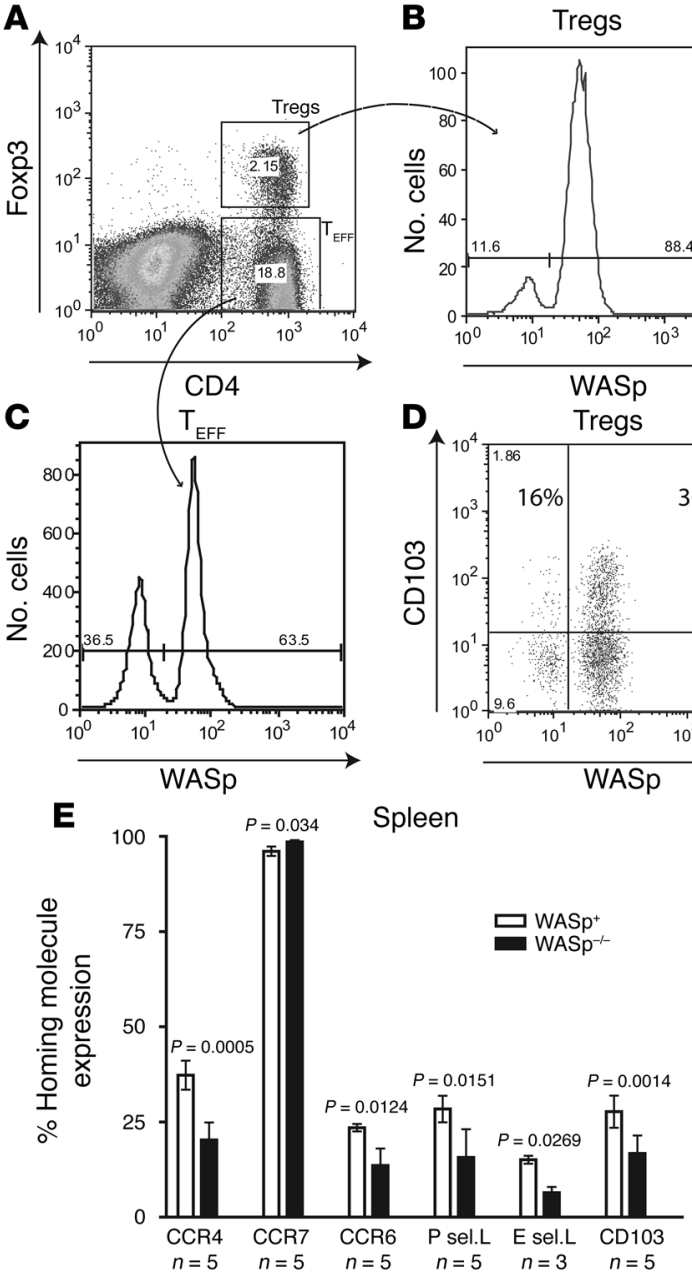

Thymic Treg development is dependent upon Foxp3 expression, cytokine signaling via the common $\gamma$ chain, and TCR-mediated positive selection (reviewed in refs. 7, 8). Interestingly, WASp appears to play a very limited role in this process. The number of thymic Tregs and their surface phenotypes were identical in WT and $\mathrm{WASp}^{-/}$mice, and Foxp3 was also expressed at normal levels in $\mathrm{WASP}^{-}$Tregs. Also, analysis of $\mathrm{X}$ inactivation in $\mathrm{WASP}^{+/-}$mice revealed no evidence for altered fitness in thymic Tregs. We also failed to identify any differences in the TCR repertoire of WASp $\mathrm{W}^{-/}$ versus WT thymic Tregs in heterozygous animals using a panel of TCR V $\beta$ antibodies (data not shown). While this analysis does not assess differences in TCR affinity, these combined observations suggest that TCR-mediated Treg selection is largely WASp independent. Further, while T cell-mediated IL-2 production is deficient in $\mathrm{WASp}^{-/-}$mice, this deficit played no role in thymic Treg production, a finding consistent with recent work (21).

Tregs comprise a stable proportion of the steady-state $\mathrm{CD} 4^{+} \mathrm{T}$ cell population. Maintenance of the Treg pool is dependent upon signals provided via the TCR, IL-2, and costimulatory molecules, including CD28/B7-1/B7-2 (refs. 7, 14, 22, and reviewed in ref. 23). Mounting evidence, including the data presented here, argue that Treg homeostasis differs in crucial ways from homeostatic cycling of naive $\mathrm{CD} 4^{+} \mathrm{CD} 25^{-} \mathrm{T}$ cells. First, compared with $\mathrm{CD} 25^{-} \mathrm{T}$ cells, Tregs appear to be significantly longer lived (15). Second, the basal proliferative response in Tregs is 2- to 5-fold greater

\section{Figure 8}

WASp $^{-1-}$ Tregs demonstrate decreased homing receptor expression relative to WT Tregs. Female WASp ${ }^{+-}$heterozygous mice (C57BL/6 strain) were analyzed for expression of CCR4, CCR6, CCR7, P selectin and $E$ selectin ligands, and CD103 in WASp+ versus WASp- Tregs in different tissues (spleen, peripheral LNs, mesenteric LNs, peritoneal fluid). (A) Total lymphocytes were stained with CD4, Foxp3, and WASp to identify Tregs (CD4+Foxp3 ${ }^{+}$, upper gate) or CD4 $\mathrm{T}_{\mathrm{EFF}}$ (CD4+Foxp3-', lower gate) cells. (B) Histogram of relative WASp expression in Tregs. (C) Histogram of WASp expression in the CD4 T $\mathrm{T}_{\mathrm{EFF}}$ compartment. (D) Example of the relative percentage of splenic WASp- versus WASp ${ }^{+}$ Tregs that coexpress CD103. (E) Mean \pm SD for relative percentage of splenic Tregs coexpressing specific adhesion/homing receptors and WASp (with $P$ values based upon paired 2-tailed Student's $t$ test). Similar data were obtained from other tissues. P sel. L, P selectin ligand; E sel. L, E selectin ligand.

than that in $\mathrm{CD}^{+} \mathrm{CD} 25^{-} \mathrm{T}$ cells, and more that $80 \%$ of splenic Tregs undergo multiple cell divisions within 30 days of transfer into nonlymphopenic hosts $(14,15)$. Third, in vivo proliferating Tregs in unimmunized, healthy animals acquire a distinct activated cell surface phenotype that includes downmodulation of CD62L and upregulation of activation markers (CD44, CD69, GITR, CD134/OX40, CD122/IL-2R $\beta$, and others) (15) and nonlymphoid tissue homing receptors, including CD103 (18). Thus, the cycling CD44 high Tregs and $\mathrm{CD} 103^{+}$Treg populations largely overlap. Together, these observations suggest that Treg homeostasis is mediated by encounters with cognate self antigen presented within the draining LNs or spleen and that this promotes expression of activation markers and receptors essential for homing and tissue entry. This activated Treg population mediates dominant tolerance via at least 3 alternative means: inhibition of the priming of colocalized naive $\mathrm{T}$ cells in the secondary lymphoid tissues, entry into germinal centers and modulation of $\mathrm{B}$ cell activation, and suppression of activated effector $T$ cells within nonlymphatic tissue sites. In contrast with this scenario, homeostatic cycling of naive $\mathrm{CD} 4^{+} \mathrm{CD} 25^{-} \mathrm{T}$ cells proceeds in the absence of costimulatory molecules and does not lead to a stable alteration in activation markers or tissue tropism.

Our combined findings support the conclusion that WASp is essential for homeostatic Treg activation and suggest, but do not prove, that WASp is required for optimal self-antigen-driven proliferation in vivo. Interestingly, WAS $\mathrm{W}^{-/-}$Tregs exhibited normal suppressive activity against $\mathrm{WASp}^{-/-}$effector $\mathrm{T}$ cells and only modestly reduced suppressive activity against WT effector T cells. Because thymic, naive, and activated Tregs behave similarly in vitro $(15,18)$, it is perhaps not surprising that this assay failed to identify defects secondary to alterations in the relative number of activated Tregs in WASp $\mathrm{W}^{-/-}$animals. In contrast with our in vitro findings, WASp function is clearly required for peripheral Treg survival and/or expansion. WASp functions to facilitate efficient T cell:APC synapse formation and sustained TCR signaling. Thus, our data suggest that WASp is required for efficient responses to cognate self antigen presented in a physiologic context in vivo. Further, while alterations in APC function might also limit Treg activation in $\mathrm{WASp}^{-/}$mice, our adoptive transfer data in $s f$ recipients demonstrate that cell-intrinsic defects are sufficient to abrogate $\mathrm{WASp}^{-/}$Tregs function in vivo.

Expression of CD103, P selectin and E selectin ligands, $\alpha 4 \beta 7$, CCR4, and CCR6 were all reduced in $\mathrm{WASp}^{-/-}$Tregs. In addition, migration of $\mathrm{WASp}^{-/-} \mathrm{T}$ cells is impaired due to their defects in 
cytoskeletal rearrangement $(24,25)$. Together, these defects are predicted to limit the capacity of $\mathrm{WASp}^{-/-}$Tregs to enter and function within inflamed tissue. Consistent with this, nearly all tissueresident Tregs in heterozygous mice were $\mathrm{WASp}^{+}$(data not shown). Defective Treg activation and migration may play an important role in the spontaneous and radiation-induced inflammatory colitis present in $\mathrm{WASp}^{-/-}$mice. Also, recent work indicates that activated Tregs can enter B cell follicles in a chemokine-dependent fashion and modulate B cell activation (26-28). Thus, the striking increase in anti-DNA antibody levels in $\mathrm{WASp}^{-/-}$animals may derive from a failure to regulate effector $\mathrm{T}$ cell activation and inefficient Treg homing to germinal centers and modulation of $\mathrm{B}$ cell activation. As BCR signaling is intact in WASp $\mathrm{W}^{-/-} \mathrm{B}$ cells $(9,10)$, loss of dominant tolerance directed toward activated $\mathrm{B}$ cells may have an enhanced phenotype in this context. Further studies are required to test this possibility and to determine whether restoration of WT Tregs alone is sufficient to abrogate autoimmunity in $\mathrm{WASp}^{-/-}$animals.

Although Tregs cannot produce IL-2, their activation is tightly linked to IL-2 produced by activated $\mathrm{CD} 4^{+} \mathrm{CD} 25^{-}$effector $\mathrm{T}$ cells, tying Treg responses to the inflammatory signals they modulate. While dispensable for Treg production and in vitro Treg activity, IL-2 provided in trans orchestrates a nonredundant growth and survival program in peripheral Tregs (21). Although WASp $\mathrm{W}^{-/-}$effector T cells exhibit defects in IL-2 production, restoring IL-2-producing capacity with normal effector $\mathrm{T}$ cells appears to be insufficient to rescue the peripheral expansion of $\mathrm{WASp}^{-/}$Tregs. This interpretation is consistent with the inability of WASp $\mathrm{W}^{-/}$- Tregs to compete effectively with WT cells in either heterozygous mice or Treg-deficient $s f$ hosts. Defects in IL-2 production may, however, accentuate the competitive disadvantage of $\mathrm{WASp}^{-/-}$Tregs in $\mathrm{WASp}^{-/-}$mice and in human patients. As naive and activated Foxp $3^{-} \mathrm{T}$ cells rely on survival signals distinct from IL-2, this difference may further contribute to the overall reduction in peripheral Treg versus effector $\mathrm{T}$ cell pool size observed in $\mathrm{WASp}^{-/}$mice.

While $\mathrm{WASp}^{-/-}$mice exhibit a marked defect in Treg activation and function, the autoimmune features of $\mathrm{WASp}^{-/-}$mice and WAS patients are less severe than those present in either Foxp3 mutant mice or humans. Previous studies of WASp function may provide insight into these differences. First, concurrent defects in effector $T$ cell activation may offset deficient Treg function by limiting basal self reactivity, expansion, or survival of the effector $\mathrm{T}$ cell pool. Second, the initial events driving effector $\mathrm{T}$ cell activation may also be blunted due to intrinsic alterations in APC function. Third, altered $T$ helper or follicular dendritic cell function might limit the capacity of activated B cells to generate high affinity autoantibodies.

Human WAS carriers exhibit nonrandom $\mathrm{X}$ inactivation in hematopoietic lineages, including Tregs, due to a competitive advantage for WASp ${ }^{+}$hematopoietic stem cells (2). This effect normally precludes any analysis of the relative competitive advantage for WASp function within human Tregs. However, identification of a WAS patient with a revertant mutation presumably affecting a lymphoid stem cell allowed us to directly demonstrate relative selective advantage for WASp-expressing Tregs in humans. At present, our findings are insufficient to directly link this improvement in competitive fitness with the clinical improvement in this individual patient. However, in light of our data in $\mathrm{WASp}^{-/-}$animals, these observations strongly sug- gest that alterations in Treg function may explain the high frequency of autoimmunity in WAS patients. Notably, 2 previous groups have identified WAS pedigrees with revertant mutations in lymphoid progenitors (29-31). Evaluation of WASp expression within Tregs in these other pedigrees and correlation of these data with autoimmune manifestations and analyses of the TCR repertoire of expanded Tregs in the patient described here and by others should provide important additional insight into the events mediating homeostatic Treg activation.

\section{Methods}

Mouse strains. The 129SvEv WASp ${ }^{-/-}$and sf/C57BL/6 mice were obtained from The Jackson Laboratory. All studies were performed using the $129 \mathrm{SvEv}$ WASp $^{-/-}$strain (9) and a C57BL/ $6 \mathrm{WASp}^{-/-}$strain generated by backcrossing with $\mathrm{C} 57 \mathrm{BL} / 6$ mice for $6-10$ generations as well as WT controls. Mice were maintained in specific pathogen-free facilities of Seattle Children's Hospital or the Benaroya Research Institute and handled according to $\mathrm{NIH}$ and institutional guidelines. All experiments were approved by either the Animal Care and Use Committee of Children's Hospital and Regional Medical Center or the Benaroya Research Institute.

Cells and reagents. Single-cell suspensions were prepared form lymphoid tissues (thymus, spleen, and peripheral and mesenteric LNs). Peyer patches were excised from the intestine, disrupted into single-cell suspension, and filtered through $100-\mu \mathrm{m}$ nytex for lymphocytes. Peritoneal and bronchoalveolar fluids were collected after lavage with cold sterile PBS. Erythrocytes were depleted by lysis with $\mathrm{NH}_{4} \mathrm{CL}_{2}$ solution. Murine cells were cultured in RPMI with 10\% FCS plus supplement (glutamine, 2-mercaptoethanol, penicillin and streptomycin, and $10 \mathrm{mM}$ HEPES). Peripheral blood was obtained from WAS patients, carriers, and controls following informed consent obtained according to the Institutional Review Board guidelines of Children's Hospital of Seattle. Human peripheral blood mononuclear cells were isolated using Ficoll-Hypaque (Amersham Pharmacia) gradient centrifugation as previously described. Platelets were separated by low-speed centrifugation.

Flow cytometry. For cell-surface staining, $10^{6}$ cells per sample were incubated with various antibodies in staining buffer (PBS and 3\% FCS) for 15 minutes on ice. Anti-murine antibodies included CD25 (PC61.5), CD8 (53-6.7), CD62L (MEL-14), CD3 (145-2C11), Gr-1 (RB6-8C5), CD103 (2E7), and Foxp3 (FJK-165) from eBioscience; and CD4 (RM4-5), CD44 (IM7), CD69 (H1.2F3), and CD11b (M1/70) from BD Biosciences - Pharmingen; and FOXP3 (259D) from BioLegend. Anti-human antibodies included FOXP3 (PCH101), CD27 (O323), and CD62L (Dreg56) from eBioscience; CD4 (RPA-T4), CD27 (M-T271), and CD45RA (HI100 and L48) from BD Biosciences - Pharmingen; and CD4 (13B8.2) from Beckman Coulter. Chemokine-IgG3 fusion proteins were used for flow cytometry of CCR4, CCR6, and CCR7 expression as previously described (32). To assess binding of $\mathrm{CD} 4^{+} \mathrm{T}$ cells to $\mathrm{P}$ selectin and $\mathrm{E}$ selectin, cells were sequentially incubated in either a $\mathrm{P}$ selectin or E selectin human IgM fusion protein (provided by J. Lowe, Case Western Reserve University, Cleveland, Ohio, USA) produced in COS-7 cells as previously described (33); this was followed by biotinylated goat anti-human IgM (Jackson ImmunoResearch Laboratories Inc.) and streptavidin-PE (eBioscience). Murine and human Foxp3 antibody-staining reagents were used according to eBioscience or BioLegend protocols, respectively. Purified polyclonal rabbit anti-WASp antibody was generated as previously described (34), and intracellular staining was performed according to instructions for the BD Biosciences - Pharmingen Fixation/Permeabilization Solution Kit, using FITC-conjugated anti-rabbit IgG secondary antibodies (Southern Biotechnology Associates) or Cy5- or PE-conjugated anti-rabbit IgG secondary antibodies (Jackson ImmunoResearch 
Laboratories Inc.). Data were acquired on a FACSCalibur or an LSR II flow cytometer (BD Biosciences) and analyzed using FlowJo for Windows version 7.1 or FlowJo for Mac version 8.2 (Tree Star Inc.).

In vitro suppression assays. For CSFE assays, $\mathrm{CD} 4^{+} \mathrm{CD} 25^{-}$cells (effector $\mathrm{T}$ cells) and $\mathrm{CD}^{+} \mathrm{CD} 25^{+}$cells (Tregs) cells were isolated from spleen and LNs of WT or WAS $\mathrm{P}^{-/-}$mice via CD4- isolation kit (Dynal); this was followed by separation of $\mathrm{CD} 25^{+}$and $\mathrm{CD} 25^{-}$fractions by CD25-PE plus anti-PE microbeads and fractionation via magnetic column (Miltenyi Biotech). CD4- spleen cells from WT mice were irradiated at 50 Gy and used as APCs in all cultures. Effector $\mathrm{T}$ cells were incubated for 9 minutes at $37^{\circ} \mathrm{C}$ in $0.8 \mu \mathrm{M}$ CFSE in PBS, washed with $100 \%$ FBS, resuspended in complete DMEM, and cultured as described in the Figure 5 legend. For thymidine assays, purified $\mathrm{CD}^{+}$cells were stained with $\mathrm{CD} 4$ and $\mathrm{CD} 25$ and FACS sorted (FACSAria cell sorter; BD Biosciences). WT CD4 $4^{+} \mathrm{CD} 25^{-}\left(5 \times 10^{4}\right)$ target cells and increasing numbers of WT versus $\mathrm{WASp}^{-/-} \mathrm{CD} 4^{+} \mathrm{CD} 25^{+}$ Tregs $\left(1.25 \times 10^{4}\right.$ to $\left.5 \times 10^{4}\right)$ or $\mathrm{CD}^{+} \mathrm{CD} 25^{-}$cells were added to achieve the correct Treg/target cell ratio in cultures also containing irradiated APCs. Cultures were stimulated with anti-CD3 for 72 hours. ${ }^{3} \mathrm{H}$-thymidine $(1 \mu \mathrm{Ci})$ was added per well during the last 8 hours of culture, and thymidine incorporation was measured in triplicate wells using a FilterMate Cell Harvester and TopCount Scintillation Counter (PerkinElmer).

ELISA assays. For detection of anti-dsDNA and anti-ssDNA antibodies, 96-well Immuno-Plates (Nunc) were coated with $0.01 \%$ poly-L-lysine solution in PBS (Sigma-Aldrich) and then with $100 \mu \mathrm{g} / \mathrm{ml}$ salmon sperm dsDNA, denatured salmon sperm ssDNA (Invitrogen), or histone from calf thymus (Sigma-Aldrich). After blocking with $0.5 \%$ BSA/PBS, serially diluted serum samples in $0.05 \%$ Tween $20 / 0.5 \%$ BSA/PBS were added to the plates in triplicate. Plates were washed with $0.05 \%$ Tween $20 / \mathrm{PBS}$ using a plate washer (SkanWasher 400; Molecular Devices), and goat anti-mouse IgG HRP (Southern Biotechnology Associates) diluted at 1:2,000 into $0.05 \%$ Tween $20 / 0.5 \%$ BSA $/ 1 \%$ goat serum/PBS was added to each well. Peroxidase reactions were developed using BD Biosciences OptEIA TMB substrate and stopped with $2 \mathrm{~N} \mathrm{H}_{2} \mathrm{SO}_{4}$. OD405 was read using Victor 3 multilabel microplate counter (PerkinElmer).The OD readings from 3 wells were averaged for each serum sample.

Murine BM transplants and adoptive transfer purified Tregs. For mixed BM chimera analysis, WT and WASp $\mathrm{W}^{-/-} \mathrm{BM}$ was isolated, rbc were lysed, and $10^{7}$ total BM cells (containing 1:3 ratio WT and KO cells) were transplanted via intravenous injection into lethally irradiated $(10.5 \mathrm{~Gy}) \mathrm{WASp}^{-/-}$recipient animals. Peripheral blood was periodically analyzed for WASp expression in different hematopoietic lineages. For neonatal transfers, $\mathrm{CD} 4^{+} \mathrm{CD} 25^{+}$Tregs (>85\% purity in all experiments) were isolated from the spleen and LNs of
8- to 12-week-old WT and WASp ${ }^{-/-}$mice (C57BL/6 strain), as described for the CFSE assay. A minimum of $10^{6}$ Tregs (WT, WASp ${ }^{-/}$, or a 50:50 mix of WT and WASp $\mathrm{W}^{-/}$cells) was transferred in $20 \mu \mathrm{l}$ PBS via i.p. injection into male neonatal $s f$ mice more than 3 days old. Mice were monitored for $s f$ phenotype, bled to measure donor chimerism (via staining for Ly5.1/Ly5.2, $\mathrm{CD} 4$, and Foxp3) starting at 14 days, and sacrificed between 30 and 50 days after transfer for full histological and phenotypic analysis.

Tissue histology. Tissues were immersion fixed in $10 \%$ neutral buffered formalin, processed into paraffin, and stained with H\&E, PAS, or periodic acid silver methenamine by standard protocols. Immunofluorescence was done on acetone-fixed frozen sections, as previously described (35).

Statistics. A paired 2-tailed Student's $t$ test was used to compare the percentage of WASp $\mathrm{W}^{+}$and $\mathrm{WASp}^{-/-}$cells within the same animal (e.g., heterozygote females), and the Mann-Whitney $U$ test was utilized to compare the number of Tregs between WT and WASp $\mathrm{W}^{-/}$mice.

\section{Acknowledgments}

We thank our WAS patients and families for participation; John Lowe and Fanny Ebling for providing reagents; Yulia Ovechkina, Ellen Renner, and Qili Zhu for technical assistance; and Angel Hui for editing assistance. S. Humblet-Baron was supported by a grant from Fonds National de la Recherche Scientifique (FNRS), Belgium (Televie), and by the Leon Frederiq Fund; B. Sather by a National Cancer Institute institutional training grant (T32-CA009537); and D.J. Campbell by a career development award from the Crohn's and Colitis Foundation of America. This work was also supported in part by NIH grants R01 CA81140, R01 HD37091, R01 HL075453, R01 DK072295, R01 DK66802, and P30 DK47754; pilot funding from the US Immunodeficiency Network (USIDNET); and the DeJoriaWiskott-Aldrich Research Fund.

Received for publication June 28, 2006, and accepted in revised form November 14, 2006.

Address correspondence to: David J. Rawlings, Department of Pediatrics, Children's Hospital \& Regional Medical Center, 307 Westlake Avenue North, Suite 300, Seattle, Washington 98109, USA. Phone: (206) 987-7450; Fax: (206) 987-7310; E-mail: drawling@ u.washington.edu.

Stephanie Humblet-Baron and Blythe Sather contributed equally to this work.
1. Sullivan, K.E., Mullen, C.A., Blaese, R.M., and Winkelstein, J.A. 1994. A multiinstitutional survey of the Wiskott-Aldrich syndrome. J. Pediatr. 125:876-885.

2. Thrasher, A.J. 2002. WASp in immune-system organization and function. Nat. Rev. Immunol. 2:635-646.

3. Imai, K., et al. 2004. Clinical course of patients with WASP gene mutations. Blood. 103:456-464.

4. Dupuis-Girod, S., et al. 2003. Autoimmunity in Wiskott-Aldrich syndrome: risk factors, clinical features, and outcome in a single-center cohort of 55 patients. Pediatrics. 111:e622-e627.

5. Burns, S., Cory, G.O., Vainchenker, W., and Thrasher, A.J. 2004. Mechanisms of WASp-mediated hematologic and immunologic disease. Blood. 104:3454-3462.

6. Schurman, S.H., and Candotti, F. 2003. Autoimmunity in Wiskott-Aldrich syndrome. Curr. Opin. Rheumatol. 15:446-453.

7. Fontenot, J.D., Gavin, M.A., and Rudensky, A.Y. 2003. Foxp 3 programs the development and func- tion of CD $4+\mathrm{CD} 25+$ regulatory T cells. Nat. Immunol. 4:330-336.

8. Sakaguchi, S., and Sakaguchi, N. 2005. Regulatory $\mathrm{T}$ cells in immunologic self-tolerance and autoimmune disease. Int. Rev. Immunol. 24:211-226.

9. Snapper, S.B., et al. 1998. Wiskott-Aldrich syndrome protein-deficient mice reveal a role for WASP in T but not B cell activation. Immunity. 9:81-91.

10. Zhang, J., et al. 1999. Antigen receptor-induced activation and cytoskeletal rearrangement are impaired in Wiskott-Aldrich syndrome proteindeficient lymphocytes. J. Exp. Med. 190:1329-1342.

11. Strom, T.S., Li, X., Cunningham, J.M., and Nienhuis, A.W. 2002. Correction of the murine WiskottAldrich syndrome phenotype by hematopoietic stem cell transplantation. Blood. 99:4626-4628.

12. Borchers, A., Ansari, A.A., Hsu, T., Kono, D.H., and Gershwin, M.E. 2000. The pathogenesis of autoimmunity in New Zealand mice. Semin. Arthritis Rheum. 29:385-399.

13. Lacout, C., et al. 2003. A defect in hematopoietic stem cell migration explains the nonrandom Xchromosome inactivation in carriers of WiskottAldrich syndrome. Blood. 102:1282-1289.

14. Setoguchi, R., Hori, S., Takahashi, T., and Sakaguchi, S. 2005. Homeostatic maintenance of natural Foxp3(+) CD25(+) CD4(+) regulatory $\mathrm{T}$ cells by interleukin (IL)-2 and induction of autoimmune disease by IL-2 neutralization. J. Exp. Med. 201:723-735

15. Fisson, S., et al. 2003. Continuous activation of autoreactive CD4+CD25+ regulatory T cells in the steady state. J. Exp. Med. 198:737-746.

16. Huehn, J., and Hamann, A. 2005. Homing to suppress: address codes for Treg migration. Trends Immunol. 26:632-636.

17. Lehmann, J., et al. 2002. Expression of the integrin alpha Ebeta 7 identifies unique subsets of CD25+ as well as CD25- regulatory T cells. Proc. Natl. Acad. Sci. U. S. A. 99:13031-13036.

18. Huehn, J., et al. 2004. Developmental stage, phenotype, and migration distinguish naive- and effector/memory-like CD4+ regulatory T cells. J. Exp. 
Med. 199:303-313.

19. Siegmund, K., et al. 2005. Migration matters: regulatory T-cell compartmentalization determines suppressive activity in vivo. Blood. 106:3097-3104.

20. Suffia, I., Reckling, S.K., Salay, G., and Belkaid, Y. 2005. A role for CD103 in the retention of CD4+CD25+ Treg and control of Leishmania major infection. J. Immunol. 174:5444-5455.

21. Fontenot, J.D., Rasmussen, J.P., Gavin, M.A., and Rudensky, A.Y. 2005. A function for interleukin 2 in Foxp3-expressing regulatory T cells. Nat. Immunol. 6:1142-1151.

22. Salomon, B., et al. 2000. B7/CD28 costimulation is essential for the homeostasis of the CD4+CD25+ immunoregulatory $\mathrm{T}$ cells that control autoimmune diabetes. Immunity. 12:431-440.

23. Sakaguchi, S. 2004. Naturally arising CD4+ regulatory $\mathrm{t}$ cells for immunologic self-tolerance and negative control of immune responses. Annu. Rev. Immunol. 22:531-562.

24. Snapper, S.B., et al. 2005. WASP deficiency leads to global defects of directed leukocyte migration in vitro and in vivo. J. Leukoc. Biol. 77:993-998.

25. Westerberg, L., et al. 2005. Wiskott-Aldrich syndrome protein deficiency leads to reduced B-cell adhesion, migration, and homing, and a delayed humoral immune response. Blood. 105:1144-1152.

26. Lim, H.W., Hillsamer, P., Banham, A.H., and Kim, C.H. 2005. Cutting edge: direct suppression of B cells by CD $4+\mathrm{CD} 25+$ regulatory T cells. J. Immunol. 175:4180-4183.

27. Bystry, R.S., Aluvihare, V., Welch, K.A., Kallikourdis, M., and Betz, A.G. 2001. B cells and professional APCs recruit regulatory $\mathrm{T}$ cells via CCL4. Nat. Immunol. 2:1126-1132.

28. Fields, M.L., et al. 2005. CD4+ CD25+ regulatory $\mathrm{T}$ cells inhibit the maturation but not the initiation of an autoantibody response. J. Immunol. 175:4255-4264.

29. Wada, T., et al. 2001. Somatic mosaicism in Wiskott-Aldrich syndrome suggests in vivo reversion by a DNA slippage mechanism. Proc. Natl. Acad. Sci.
U. S. A. 98:8697-8702

30. Ariga, T., et al. 2001. Spontaneous in vivo reversion of an inherited mutation in the Wiskott-Aldrich syndrome. J. Immunol. 166:5245-5249.

31. Wada, T., et al. 2004. Multiple patients with revertant mosaicism in a single Wiskott-Aldrich syndrome family. Blood. 104:1270-1272.

32. Zhou, B., et al. 2005. Thymic stromal lymphopoietin as a key initiator of allergic airway inflammation in mice. Nat. Immunol. 6:1047-1053.

33. Knibbs, R.N., et al. 1998. Alpha(1,3)-fucosyltransferase VII-dependent synthesis of P-and E-selectin ligands on cultured T lymphoblasts. J. Immunol. 161:6305-6315.

34. Zhu, Q., et al. 1997. Wiskott-Aldrich syndrome/ $\mathrm{X}$-linked thrombocytopenia: WASP gene mutations, protein expression, and phenotype. Blood. 90:2680-2689.

35. Taneda, S., et al. 2001. Cryoglobulinemic glomerulonephritis in thymic stromal lymphopoietin transgenic mice. Am. J. Pathol. 159:2355-2369. 